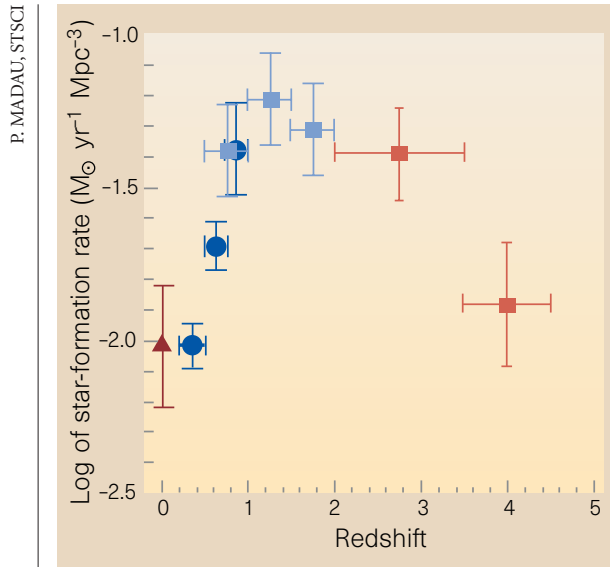

Figure 2 The star-formation history of the Universe. The formation rate is in units of solar masses per year per cubic megaparsec. Individual points come from redshift surveys which, taken together, cover $80-90 \%$ of the total age of the Universe. (Red triangle from ref. 10; dark blue circles from refs 1 and 2; light blue squares from ref. 11; orange squares from ref. 5.)

from regions within the Hubble Deep Field (M. Rowan-Robinson, Imperial College). Furthermore, simulations of distant, ultraluminous counterparts to local starburst galaxies show that some of them would resemble certain classes of peculiar galaxy detected in the Hubble Deep Field (W. Vacca, Univ. Hawaii).

But more modest dust extinction has been inferred from Lyman dropout systems (M. Pettini, Royal Greenwich Obs.; M. Dickinson, Johns Hopkins). Their spectra do not turn down strongly at short wavelengths, as would be expected from strong dust extinction. This still implies that the high-redshift tail of the star-formation history curve is too low, but only by a factor of 3-4.

There are two arguments in favour of the lesser degree of dust extinction. Increasing the curve by much more than a factor of 3-4 may result in a contradiction with existing data: the diffuse infrared background flux is close to that predicted from the present version of Madau's curve (L. Cowie, Univ. Hawaii). Also, to avoid producing more lowmass (therefore long-lived) stars than we see now, a factor of ten increase in the early starformation rate would require that star formation at the time was biased in favour of producing only massive (hence short-lived) stars. But even this rather unpleasant assumption solves only part of the problem, because high-mass stars eject metals into the intergalactic medium, and the integrated abundance of metals from Madau's curve is roughly what we see in the local Universe. One possible way out is that the metals produced by high-redshift star formation are locked away unobserved in between galaxies ${ }^{9}$.

A final curiosity is that the star-formation history curve, at least in its present form, is qualitatively similar to quasar evolution. Perhaps quasar evolution is more intimately linked to galaxy evolution than hitherto believed. If so, the star-formation history curve may help to address one of the great unsolved mysteries in astrophysics: where have all the quasars gone?

Roberto Abraham is at the Royal Greenwich

Observatory, Madingley Road, Cambridge

CB3 OEZ, UK.

1. Lilly, S. J., Le Fevre, O., Hammer, F. \& Crampton, D. Astrophys. J. 460, L1-L4 (1996).

2. Ellis, R. S., Colless, M., Broadhurst, T., Heyl, J. \& Glazebrook,
K. Mon. Not. R. Astron. Soc. 280, 235-251 (1996)

3. Cowie, L. L., Songaila, A., Hu, E. M. \& Cohen, J. D. Astron. J. 112, 834-864 (1996).

4. Steidel, C. C., Giavalisco, M., Pettini, M., Dickinson, M. E. \& Adelberger, K. Astrophys. J. 462, L17-L22 (1996).

5. Madau, P. et al. Mon. Not. R. Astron. Soc. 283, 1388-1404 (1996).

6. Giavalisco, M., Steidel, C. C. \& Macchetto, F. D. Astrophys. J. 470, 189-194 (1996)

7. Baugh, C., Cole, S. \& Frenk, C. S. 282, L27-L32 (1996).

8. Abraham, R. G. et al. Mon. Not. R. Astron. Soc. 279, L47-L52 (1996)

9. Mushotzky, R. F. \& Loewenstein, M. Astrophys. J. (in the press).

10. Gallego, J. et al. Astrophys. J. 455, L1-L4 (1995).

11. Connolly, A. J. et al. Astrophys. J. (in the press).

\title{
Potassium channels
}

\section{Dendritic shock absorbers}

\section{Rafael Yuste}

$\mathrm{M}$ ammalian neurons have dendritic trees - branched structures with complex geometries - which have mesmerized researchers for over a century. In 1891, Santiago Ramón y Cajal noticed that whereas sensory neurons point their dendrites towards the periphery, they always send their axons towards the brain. $\mathrm{He}$ suggested that a neuron receives information through its dendrites, and that it transmits this information through its axon. But exactly how the dendrites receive and integrate sensory inputs, and transfer them to the axon, is still a mystery.

On page 869 of this issue, Hoffman et al. ${ }^{1}$ report that they have found potassium channels in dendrites from hippocampal pyramidal neurons. Why is this of general importance? As the authors show, potassium channels play a key role in dendritic excitability, and they may mediate sophisticated computations required for learning.

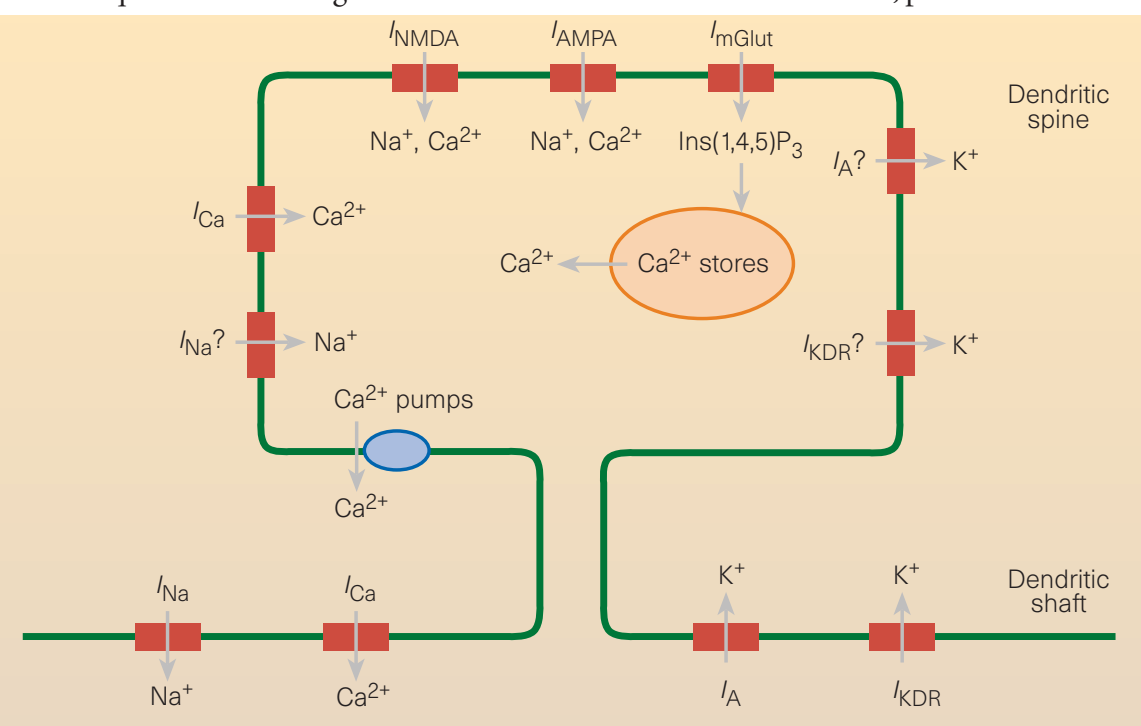

Figure 1 Known and postulated channels and receptors in dendritic spines and dendritic shafts of CA1 neurons in the hippocampus. Conductances: $I_{A}$, A-type potassium channels; $I_{\mathrm{KDR}}$, delayedrectifier potassium channels; $I_{\mathrm{Na}}$, sodium channels; $I_{\mathrm{Ca}}$ calcium channels. Glutamate receptors: $I_{\mathrm{NMDA}}, N$-methyl-D-aspartate receptor; $I_{\mathrm{AMPA}}$, aminohydroxymethylisoxalate propionic acid; $I_{\mathrm{mGlut}}$ metabotropic glutamate receptor. Ins $(1,4,5) \mathrm{P}_{3}$, inositol-1,4,5-trisphosphate. 


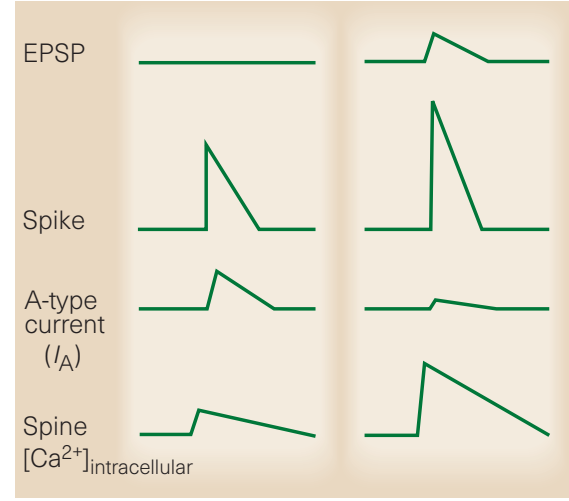

Figure 2 Hoffman et al. ${ }^{1}$ have found that potassium channels exist on dendrites of hippocampal pyramidal (CA1) neurons. They propose that the temporal coincidence of excitatory postsynaptic potentials (EPSPs) and back-propagating action potentials (spikes) results in a larger spike, because these dendritic A-type potassium channels are inactivated. The larger spike will lead to a greater influx of calcium into the dendritic spines, and this may underlie long-term potentiation - a putative cellular mechanism of learning.

maintain the resting potential, and they regulate the hyperpolarization of an action potential. So, in dendrites, potassium channels could act as 'shock absorbers', dampening the depolarization that is produced by excitatory inputs - also known as excitatory postsynaptic potentials (EPSPs) - and the effect of the sodium channels (which produce action potentials) and calcium channels. Although dendritic potassium channels have been found in turtle Purkinje cells ${ }^{7}$, until now the existence and possible function of potassium channels in mammalian dendrites had not been directly shown.

Hoffman et al. ${ }^{1}$ have done a detailed study of dendritic potassium channels in apical dendrites of CA1 pyramidal neurons from the hippocampus, and they find a conductance with physiological and pharmacological profiles corresponding to the so-called A-type potassium channels. The authors also describe lower levels of another conductance that is analogous to the delayed-rectifier potassium channel. Finding a strong A-type current in apical dendrites is consistent with previous studies using intradendritic recordings $^{8}$ and immunohistochemistry ${ }^{9}$, increasing the number of voltage-sensitive channels that are known to be present in CA1 dendrites. So, not only are dendrites active, but they may have more different types of channels than axons (Fig. 1).

But what are all of these active conductances doing? In many types of neuron, action potentials from the cell body are known to 'back-propagate' through the dendritic tree ${ }^{10}$ (although the extent of this phenomenon in vivo is still unclear $\left.{ }^{11}\right)$. This back-propagating action potential (or spike) is mediated by dendritic sodium channels.
It reaches the fine dendritic branches and spines, opening calcium channels along its way and causing large influxes of calcium into dendritic shafts and spines ${ }^{12,13}$. So, dendritic spines, which receive the EPSPs (that is, the input into the cell), can also 'hear' the spike (that is, the output of the cell), meaning that they are ideally poised to detect and compute temporal coincidences of neuronal inputs and outputs.

In neocortical pyramidal neurons, the temporal coincidence of EPSPs and backpropagating spikes produces long-term synaptic potentiation (LTP) - a cellular mechanism that may underlie learning ${ }^{14,15}$. But if the spikes reach the dendritic spines before the EPSPs occur, there is a long-lasting decrease in synaptic efficacy; that is, cellular 'forgetting' ${ }^{15}$. So, back-propagating spikes, and their exact timing with respect to the EPSPs, are required for this neuronal plasticity.

In CA1 neurons, back-propagating spikes also seem to be necessary for LTP, because LTP does not occur if sodium-channel-mediated spikes are blocked ${ }^{16,17}$. The detection of coincident EPSPs and backpropagating spikes in LTP is thought to be implemented by an influx of calcium through the NMDA ( $N$-methyl-D-aspartate) type of glutamate receptor (Fig. 1) when glutamate binds to it and the postsynaptic cell is depolarized. This calcium influx could then orchestrate long-lasting changes in synaptic efficacy. Indeed, LTP does not occur if NMDA receptors are blocked or if postsynaptic calcium is chelat$\mathrm{ed}^{14}$. In agreement with the idea that the dendritic spines detect coincident EPSPs and back-propagating spikes, when the two phenomena occur simultaneously, higher, supralinear calcium accumulates in the spines ${ }^{13}$.

Dendritic A-type currents now provide a new mechanism for this detection. Using computer simulations, Hoffman et al. ${ }^{1}$ find that dendritic A-type channels can be inactivated by the depolarization that is produced during EPSPs. This inactivation will produce a larger, full-blown, back-propagating spike, because the A-type channels will no longer dampen it (Fig. 2). Indeed, a larger backpropagating spike is measured in apical dendrites when an EPSP occurs simultaneous$\mathrm{ly}^{17}$. This larger spike could open more calcium channels in the dendritic spine, increasing the influx of calcium during coincident EPSPs and spikes. Moreover, the larger backpropagating spike would unblock the NMDA receptors more efficiently, increasing their permeability to calcium. So the temporal coincidence of the input and output of the cell would be detected by the effect of the A-type channels on the spike, and translated into a greater accumulation of calcium. But more experiments are needed to establish this interesting hypothesis.
LTP is, in fact, only one of the many functions that occur in dendrites. Dendritic potassium channels could influence other aspects of dendritic integration; for example, active dendritic conductances could shape the EPSPs. Indeed, Hoffman et al. show that dendritic sodium channels boost EPSPs, but that this boost is normally prevented by Atype potassium currents. Finally, potassium channels could also control dendritic spiking, because blocking A-type currents leads to the generation of dendritic action potentials triggered by EPSPs or an injection of current ${ }^{8}$. So the spike initiation zone, which is generally assumed to be in the axon and/or cell body, may shift to the dendrite if potassium channels are inactivated under particular physiological conditions. Dendritic potassium channels may also help us to understand why spikes are not generated in the dendrites - where EPSPs originate in spite of there being a similar density of sodium channels in dendrites and the cell body.

Potassium channels have many regulatory mechanisms. All of the putative functions of the dendritic potassium channels could be specifically regulated in different parts of the dendritic tree. For example, trains of EPSPs or IPSPs (inhibitory post-synaptic potentials), second messengers, modulatory neurotransmitters and changes in extracellular ions or in redox states of the cell could, in principle, modify the activation or inactivation kinetics of potassium channels and alter the delicate balance of active conductances in the dendrites. Now, more than ever, a detailed knowledge of the nuts and bolts of dendritic processing seems essential for a proper understanding of neuronal plasticity and function.

Rafael Yuste is in the Department of Biological

Sciences, Columbia University, 1212 Amsterdam

Avenue, New York, New York 10027, USA.

1. Hoffman, D. A., Magee, J. C., Colbert, C. M. \& Johnston, D. Nature 387, 869-875 (1997).

2. Llinás, R., Nicholson, C., Freeman, J. A. \& Hillman, D. E. Science 160, 1132-1135 (1968)

3. Spencer, W. A. \& Kandel, E. R. J. Neurophysiol. 24, 272-285 (1961).

4. Johnston, D., Magee, J. C., Colbert, C. M. \& Christie, B. R. Аnпu. Rev. Neurosci. 19, 165-186 (1996).

5. Yuste, R. \& Tank, D. W. Neuron 16, 701-716 (1996).

6. Hille, B. Ionic Channels in Excitable Membranes (Sinauer, Sunderland, MA, 1992).

7. Hounsgaard, J. \& Mitgaard, J. J. Physiol., Lond. 402, 731-749 (1988).

8. Andreasen, M. \& Lambert, J. D. C. J. Physiol., Lond. 483, 421-441 (1995).

9. Sheng, M., Tsaur, M., Jan, Y. N. \& Jan, L. Y. Neuron 9, 271-284 (1991).

10.Stuart, G. J. \& Sakmann, B. Nature 367, 69-72 (1994).

11. Svoboda, K., Denk, W., Kleinfeld, D. \& Tank, D. W. Nature 385, 161-165 (1997).

12. Spruston, N., Schiller, Y., Stuart, G. \& Sakmann, B. Science 286, 297-300 (1995).

13. Yuste, R. \& Denk, W. Nature 375, 682-684 (1995).

14. Baudry, M. \& Davis, J. L. Long-Term Potentiation Vols 1-3 (MIT Press, Cambridge, MA, 1991, 1994, 1997).

15. Markram, H., Luebke, J., Frotscher, M. \& Sakmann, B. Science 275, 213-215 (1997).

16. Scharfman, H. E. \& Sarvey, J. M. Brain Res. 331, 267-274 (1985).

17. Magee, J. C. \& Johnston, D. Science 275, 209-212 (1997). 